\title{
Global Exponential Stability of Uncertain Neural Networks with Discontinuous Lurie-type Activation and Mixed Delays
}

\author{
Sitian Qin ${ }^{\mathrm{a}}$, Qun Cheng ${ }^{\mathrm{a}}$, Guofang Chen ${ }^{\mathrm{b}, *}$ \\ ${ }^{a}$ Department of Mathematics, Harbin Institute of Technology at Weihai, Weihai 264209, \\ PR China \\ ${ }^{b}$ Department of Civil Engineering, Harbin Institute of Technology at Weihai, Weihai \\ 264209, PR China
}

\begin{abstract}
This paper deals with the problem of the global exponential stability of a class of uncertain neural networks with discontinuous Lurie-type activation and mixed delays. By establishing a new sufficient condition, we first prove the existence of the equilibrium point by using the Leary-Schauder alternative theorem. Then, by employing a new Lyapunov functional, we obtain the global exponential stability of the equilibrium point of the uncertain neural network. In the end, some comparisons and numerical examples are given to show the improvement of the conclusions in this paper.
\end{abstract}

Keywords: Neural network with Lurie-type activation and mixed delays; global exponential stability; Leary-Schauder alternative theorem; Lyapunov functional

\section{Introduction}

In recent years, neural networks have been extensively investigated since their wide applications, such as pattern recognition, associative memories, automatic control, optimization, image processing and other areas (see $[1,2$, $3,4,5,6,7,8]$ ). It is well known that the applications of neural networks heavily depend on their stability. Therefore, in order to successfully employ neural networks in applications, we should study the stability of the designed neural network in advance. However, due to the finite switching speed of the neuron amplifiers and transmissions of signals in a network, time delays are actually unavoidable in the electronic implementation. Meanwhile, in

\footnotetext{
* Corresponding author

Email addresses: qinsitian@163.com (Sitian Qin), chengqunchn@163.com (Qun Cheng), hitbri@126.com (Guofang Chen)

${ }^{1}$ This research is supported by the National Science Foundation of China (61403101) and Weihai Science and technology Development Plan Project (2013DXGJ08).
} 
both biological and artificial neural networks, the delay may create the loss of stability, since it may originate the onset of nonvanishing oscillations (see $[9,10,11,12,13,14,15,16,17,18,19,20,21,22,23,24,25])$. On the other hand, the estimation errors are unavoidable when we measure the vital data of deterministic neural networks such as the neuron fire rate and the synaptic interconnection weights. Moreover, in deterministic neural networks, the stability of neural networks can often be destroyed by its compulsory uncertainty issuing from the existence of modeling errors, external disturbance and parameter fluctuations. Hence, it is important and necessary to study the robust stability of neural networks with time delays in presence of uncertainties (see $[13,14,15,26,27])$.

In the past years, the stability of uncertain neural network has received considerable attentions, and varieties of interesting results have been presented in the literatures (see [10, 13, 14, 15, 26, 27, 28]). For example, in [10], authors investigated the problem of the existence, uniqueness and global asymptotic stability of the equilibrium point for the class of neural networks with multiple time delays and parameter uncertainties. By means of the homeomorphism theory and Lyapunov functional method, Arik in [13] studied the global asymptotic stability problem of dynamical neural networks with multiple time delays under parameter uncertainties. Guo et.al in [26] presented a systematic method for analyzing the robust stability of a class of interval neural networks with uncertain parameters and time delays. In [28], we studied the global robust exponential stability of the neural networks with possibly unbounded activation functions.

However, most of the results concerning the robust stability of neural networks are based on a common assumption that the activations are continuous or even Lipschitz continuous (see $[9,10,13,14,15,29,30]$ ). In practice, as mentioned by [31], discontinuous or non-Lipschitz neuron activation$\mathrm{s}$ have been introduced into neural network systems due to their theoretical and practical significance in recent years. Nowadays, more and more scholars observe the importance of neural network with discontinuous activations (see $[18,32,33,34,35,35,36,37])$. For example, Qin et. al. in [18] studied the global exponential stability and global convergence in finite time of neural networks with discontinuous activations. In [38], a novel class of Cohen-Grossberg neural networks with delays and inverse Hölder neuron activation functions are presented. In [37], authors integrated a class of delayed neural networks with discontinuous activations by means of the Leray-Schauder theorem and Viability theorem. In [23], authors investigated the global dynamics of equilibrium point for delayed competitive neural networks with different time scales and discontinuous activations.

Inspired by previous studies, in this paper, we will study the existence, global exponential stability of uncertain neural networks with discontinuous Lurie-type activation and mixed delays. The remainder of this paper is arranged as follows. In section 2, we state some preliminaries including some necessary definitions and lemmas. Our main results are contained in Sections 3 and 4, where the sufficient conditions are given to guarantee the existence and global exponential stability of equilibrium for neural networks in this paper. Section 5 present 
some illustrative numerical examples to verify our results.

Notation Given the column vector $x=\left(x_{1}, x_{2}, \ldots, x_{n}\right)^{T}$, where the superscript $T$ is the transpose operator, and $\|x\|:=\left(\sum_{i=1}^{n} x_{i}^{2}\right)^{\frac{1}{2}}$. Let $A=\left(a_{i j}\right) \in \mathbb{R}^{n \times n}$ and define $\|A\|=\sqrt{\lambda_{M}\left(A^{T} A\right)}$, where $\lambda_{M}(A)$ stands for the operation of taking the maximum eigenvalue of $A$. $I \in \mathbb{R}^{n \times n}$ is the $n \times n$ identity matrix. For a real symmetric matrix $\mathrm{A}, A<0$ means that $A$ is negative definite .

\section{Preliminaries}

In this section, we will introduce some definitions and lemmas, which will be used in the remainder of this paper. The neural network we consider in this paper is described as follows,

$$
\begin{aligned}
\frac{d x_{i}(t)}{d t}= & -d_{i} x_{i}(t)+\sum_{j=1}^{n} a_{i j} g_{j}\left(x_{j}(t)\right)+\sum_{j=1}^{n} b_{i j} g_{j}\left(x_{j}\left(t-\tau_{j}\right)\right) \\
& +\sum_{j=1}^{n} c_{i j} \int_{t-h_{j}}^{t} g_{j}\left(x_{j}(s)\right) d s+u_{i},
\end{aligned}
$$

or equivalently,

$$
\begin{aligned}
\dot{x}(t)= & -D x(t)+A g(x(t))+B g(x(t-\tau)) \\
& +C \int_{t-h}^{t} g(x(s)) d s+U,
\end{aligned}
$$

where $x=\left(x_{1}, x_{2}, \ldots, x_{n}\right)^{T} \in \mathbb{R}^{n}$ denotes the state of the neurons; $D=$ $\operatorname{diag}\left(d_{1}, d_{2}, \ldots, d_{n}\right)^{T} \in \mathbb{R}^{n}$ is a positive diagonal matrix; $A=\left(a_{i j}\right)_{n \times n}, B=$ $\left(b_{i j}\right)_{n \times n}, C=\left(c_{i j}\right)_{n \times n}$, are the $n \times n$ real connection weight matrices representing the weighting coefficients of the neurons; $g=\left(g_{1}\left(x_{1}\right), g_{2}\left(x_{2}\right), \ldots, g_{n}\left(x_{n}\right)\right)^{T} \in$ $\mathbb{R}^{n}$ represents the neuron activations.

In this paper, we will study the neural network (1) with general activation functions. That is, the activation functions are only assumed to be nonincreasing. For convenience, we define

$$
\mathcal{M} \triangleq\left\{\phi: \mathbb{R} \rightarrow \mathbb{R} \mid s \phi(s) \geq 0, \text { and } D^{+} \phi(s) \geq 0, s \in \mathbb{R}\right\} .
$$

For any $\phi \in \mathcal{M}$, we define

$$
K[\phi(t)]=\left[\phi\left(t^{-}\right), \phi\left(t^{+}\right)\right]
$$

In this paper, we always assume the following assumptions hold,

$\left(\mathbf{A}_{\mathbf{1}}\right)$ The activation function of neural network (1) satisfies the Lurie-type condition. That is, $g_{i} \in \mathcal{M}$, and there exists $k_{i}>0$ such that

$$
x_{i} g_{i}\left(x_{i}\right) \leq k_{i} x_{i}^{2}
$$


for any $x_{i} \in \mathbb{R}$. stants.

Here, the constants $k_{i}, i=1,2, \ldots, n$, are generally called to be Lurie con-

$\left(\mathbf{A}_{2}\right)$ The parameters $A=\left(a_{i j}\right)_{n \times n}, B=\left(b_{i j}\right)_{n \times n}, C=\left(c_{i j}\right)_{n \times n}, D=$ $\operatorname{diag}\left(d_{i}\right)$ are assumed to be intervalised as follows,

$$
\begin{aligned}
& D_{I}:=\left\{D=\operatorname{diag}\left(d_{i}\right): 0 \leq \underline{D} \leq D \leq \bar{D}, \text { i.e., }, \underline{d_{i}} \leq d_{i} \leq \overline{d_{i}}, \forall i\right\} \\
& A_{I}:=\left\{A=\left(a_{i j}\right): \underline{A} \leq A \leq \bar{A}, \text { i.e., } a_{i j} \leq a_{i j} \leq \overline{a_{i j}}, \forall i, j\right\} \\
& B_{I}:=\left\{B=\left(b_{i j}\right): \underline{B} \leq B \leq \bar{B}, \text { i.e., } \underline{b_{i j}} \leq b_{i j} \leq \overline{b_{i j}}, \forall i, j\right\}
\end{aligned}
$$

Next lemma is Leary-Schauder alternative theorem, which plays an important role in prove the existence of the equilibrium point of the neural network (2).

Lemma 2.1. [39] If $X$ is a Banach space, $C \subseteq X$ is nonempty convex with $0 \in C$ and $G: C \rightarrow P_{k(c)}(C)$ is an USC multifunction which maps bounded sets into relatively compact sets, then one of the following statements is true,

(1) the set $\Gamma=\{x \in C: x \in \lambda G(x), \lambda \in(0,1)\}$ is unbounded;

(2) the $G(\cdot)$ has a fixed point in $C$, i.e., there exists $x \in C$ such that $x \in G(x)$.

Definition 2.1. $x^{*}$ is said to be an equilibrium point of neural network (2) if there exists $\gamma^{*} \in K\left[g\left(x^{*}\right)\right]$ such that

$$
-D x^{*}+(A+B) \gamma^{*}+C H \gamma^{*}+U=0,
$$

where $H=\operatorname{diag}\left(h_{i}\right), i=1,2, \ldots, n$. And $\gamma^{*}$ is said to be an output equilibrium point of system (2) corresponding to $x^{*}$.

Definition 2.2. [35] For any continuous function $\phi:[-\tau, 0] \rightarrow \mathbb{R}^{n}$ and any measurable selection $\psi:[-\tau, 0] \rightarrow \mathbb{R}^{n}$, such that $\psi(s) \in K[g(\phi(s))]$ for a.a. $s \in[-\tau, 0]$, by an initial value problem(IVP) associated to (2) with initial condition $(\phi, \psi)$, we mean the following problem: find a couple of functions $[x, \gamma]:[-\tau, T) \rightarrow \mathbb{R}^{n} \times \mathbb{R}^{n}$, such that $x$ is a solution of $(2)$ on $[-\tau, T)$ for some $T>0, \gamma$ is an output associated to $x$, and

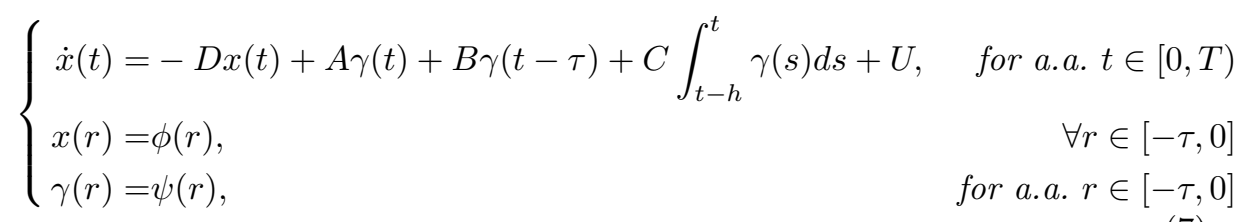

Lemma 2.2. [40] Let $x=\left(x_{1}, x_{2}, \ldots, x_{n}\right)^{T} \in \mathbb{R}^{n}$, if

$$
A \in A_{I}:=\left\{A=\left(a_{i j}\right): 0 \leq \underline{A} \leq A \leq \bar{A}, \text { i.e., } 0 \leq \underline{a_{i j}} \leq a_{i j} \leq \overline{a_{i j}}, \forall i, j\right\}
$$


then, for any positive diagonal matrix $P$, the following inequality holds:

$$
x^{T}\left(P A+A^{T} P\right) x \leq x^{T}\left(P A^{*}+A^{* T} P+\left\|P A_{*}+A_{*}^{T} P\right\|_{2} I\right) x,
$$

where $A^{*}=\frac{1}{2}(\underline{A}+\bar{A}), A_{*}=\frac{1}{2}(\bar{A}-\underline{A})$.

Lemma 2.3. [28] Let $x=\left(x_{1}, x_{2}, \ldots, x_{n}\right)^{T} \in \mathbb{R}^{n}$, if

$$
B \in B_{I}:=\left\{B=\left(b_{i j}\right): 0 \leq \underline{B} \leq B \leq \bar{B}, i . e ., 0 \leq \underline{b_{i j}} \leq b_{i j} \leq \overline{b_{i j}}, \forall i, j\right\},
$$

then, the following inequality holds:

$$
\|B\|_{2} \leq \mathrm{b},
$$

where $\mathrm{b}=\min \left\{\left\|B^{*}\right\|_{2}+\left\|B_{*}\right\|_{2},\|\hat{B}\|_{2}, \sqrt{\left\|B^{*}\right\|_{2}^{2}+\left\|B_{*}\right\|_{2}^{2}+2\left\|B_{*}^{T}\left|B^{*}\right|\right\|_{2}}\right\}$, $B^{*}=\frac{1}{2}(\underline{B}+\bar{B})$, and $B_{*}=\frac{1}{2}(\bar{B}-\underline{B})$. Define $\hat{B}=\hat{b_{i j}}=\max \left\{\left|\underline{b}_{i j}\right|,\left|\bar{b}_{i j}\right|\right\}$.

Lemma 2.4. Let $H<0$. Given any $x, y \in \mathbb{R}^{n}$, and matrix $A \in \mathbb{R}^{n \times n}$, the following inequality holds:

$$
x^{T} A y \leq \frac{1}{2 \epsilon} x^{T} A A^{T} x+\frac{\epsilon}{2} y^{T} y .
$$

Lemma 2.5. [41] For any constant matrix $\chi \in \mathbb{R}^{n \times n}, \chi=\chi^{T}$, scalar $v>0$, vector function $F:[0, v] \rightarrow \mathbb{R}^{n}$ such that the integrations concerned are welldefined, then,

$$
v \int_{v}^{0} F^{T}(s) \chi F(s) d s \geq\left(\int_{v}^{0} F(s) d s\right)^{T} \chi\left(\int_{v}^{0} F(s) d s\right) .
$$

\section{Existence of the equilibrium point of neural network (2)}

In this section, we will study the existence of an equilibrium for neural network (2) by the Leray-Schauder alternative theorem.

Theorem 3.1. Suppose the assumptions $\left(\mathbf{A}_{\mathbf{1}}, \mathbf{A}_{\mathbf{2}}\right)$ hold, and the network parameters satisfy (5). Then, neural network (2) has an equilibrium point, if there exists a positive diagonal matrix $P=\operatorname{diag}\left\{p_{i}\right\}$ such that

$$
\Phi_{1}=\left(P A^{*}+A^{* T} P+\left\|P A_{*}+A_{*}^{T} P\right\|_{2} I\right)+2\|P\|_{2} \mathrm{~b}+\|P C\|_{2}^{2} I+H^{2}<0,
$$

where $\mathrm{b}$ is defined in Lemma 2.3.

Proof. Let $F(x)=D^{-1}(A+B) K[g(x)]+D^{-1} C H K[g(x)]+D^{-1} U$. Then, $x^{*}$ is a fixed point of $F$ if and only if $x^{*}$ is an equilibrium point of the neural network (2). By the Leray-Schauder alternative Theorem, to solve the fixed point problem $x^{*} \in F\left(x^{*}\right)$, it is sufficient to show that the set

$$
\Gamma=\left\{x \in \mathbb{R}^{n}: x \in \theta F(x), \theta \in(0,1)\right\}
$$


is bounded. Next, we will prove there exists $R>0$, such that $\Gamma \subseteq B(0, R)$, which means that $\Gamma$ is bounded. Let

$$
\begin{aligned}
F(x, \theta) & =D[-x+\theta F(x)] \\
& =-D x+\theta(A+B) K[g(x)]+\theta C H K[g(x)]+\theta U .
\end{aligned}
$$

For any $y \in F(x, \theta)$, there must exist $\gamma \in K[g(x)]$ such that

$$
y=-D x+\theta(A+B) \gamma+C H \gamma+\theta U \text {. }
$$

Multiplying both sides of the equation above by $2 \gamma^{T} P$, we have

$$
\begin{aligned}
2 \gamma^{T} P y= & 2 \gamma^{T} P(-D x+\theta(A+B) \gamma+\theta C H \gamma+\theta U) \\
= & -2 \gamma^{T} P D x+2 \theta \gamma^{T} P(A+B) \gamma+2 \theta \gamma^{T} P C H \gamma+2 \theta \gamma^{T} P U \\
= & -2 \gamma^{T} P D x+2 \theta \gamma^{T} P A \gamma+2 \theta \gamma^{T} P B \gamma+2 \theta \gamma^{T} P C H \gamma+2 \theta \gamma^{T} P U \\
= & -2 \gamma^{T} P D x+\theta \gamma^{T}\left(P A+A^{T} P\right) \gamma+2 \theta \gamma^{T} P B \gamma+2 \theta \gamma^{T} P C H \gamma \\
& +2 \theta \gamma^{T} P U .
\end{aligned}
$$

According to the assumption $\left(\mathbf{A}_{\mathbf{1}}\right)$, we have

$$
-2 \gamma^{T} P D x \leq-2 \gamma^{T} P \underline{D} x=-2\left|\gamma^{T}\right| P \underline{D}|x| \leq-2 \theta\left|\gamma^{T}\right| P \underline{D}|x| .
$$

From Lemma 2.2, we can get

$$
\theta \gamma^{T}\left(P A+A^{T} P\right) \gamma \leq \theta \gamma^{T}\left(P A^{*}+A^{* T} P+\left\|P A_{*}+A_{*}^{T} P\right\|_{2} I\right) \gamma,
$$

where $K=\operatorname{diag}\left\{k_{i}\right\}$. It is clear that,

$$
2 \theta \gamma^{T} P B \gamma \leq 2 \theta \gamma^{T} P B \gamma \leq 2 \theta \gamma^{T}\|P B\|_{2} \gamma \leq 2 \theta \gamma^{T}\|P\|_{2}\|B\|_{2} \gamma .
$$

From Lemma 2.2 and (13),

$$
2 \theta x^{T} P B \gamma \leq 2 \theta x^{T}\|P\|_{2} \mathrm{~b} \gamma .
$$

Then, by (12),(14), we can get

$$
\begin{aligned}
& 2 \theta \gamma^{T} P(A+B) \gamma+2 \theta \gamma^{T} P C H \gamma \\
\leq & \theta \gamma^{T}\left(P A^{*}+A^{* T} P+\left\|P A_{*}+A_{*}^{T} P\right\|_{2} I+2\|P\|_{2} \mathrm{~b}\right) \gamma+\theta \gamma^{T}\|P C\|_{2}^{2} \gamma+\theta \gamma^{T} H^{2} \gamma \\
= & \theta \gamma^{T} \Phi_{1} \gamma \\
\leq & 0
\end{aligned}
$$

Asserting (11) and (15) into (10) results in:

$$
\begin{aligned}
2 \gamma^{T} P y & \leq-2 \gamma^{T} P D x+2 \theta \gamma^{T} P U \\
& =2 \sum_{i=1}^{n} P_{i} \gamma_{i}\left(-D_{i} x_{i}+U_{i}\right)
\end{aligned}
$$


Then, similar to [28], we can construct an open convex bounded set $\Omega$, such that $2 \gamma^{T} P y<0$ for all $x \in \Omega$. Hence, $\Gamma \subseteq \Omega$ is bounded. Invoking Lemma 2.1 , there exists $x^{*} \in \mathbb{R}^{n}$ such that $x^{*} \in F\left(x^{*}\right)$. Of course, $x^{*}$ is an equilibrium point of neural network (2).

\section{Stability analysis}

In this section, we will show that the equilibrium point of neural network (2) is globally robustly exponentially stable based on assumptions in Theorem 3.1. In order to simplify the proof, we need to shift the equilibrium point $x^{*}$ of neural network (2) to the origin as follows,

$$
z(\cdot)=x(\cdot)-x^{*} .
$$

From Definition 2.1, there exists $\gamma^{*} \in K\left[g\left(x^{*}\right)\right]$ such that

$$
-D x^{*}+(A+B) \gamma^{*}+C H g\left(x^{*}\right)+U=0,
$$

Then the neural network (2) is equivalent to the following neural network,

$$
\dot{z}(t)=-D z(t)+A \tilde{G}(z(t))+B \tilde{G}(z(t-\tau))+C \int_{t-h}^{t} \tilde{G}(z(s)) d s,
$$

where $\tilde{G}_{i}(z)_{i}=g_{i}\left(z_{i}+x_{i}^{*}\right)-\gamma_{i}^{*}$. Obviously, $O(0,0, \ldots, 0)$ is an equilibrium point of neural network (18). By Definition 2.2,z(t) is a solution of the neural network (18), if there exists $\tilde{\gamma}(z(t)) \in K[\tilde{G}(z(t))]$ such that

$$
\dot{z}(t)=-D z(t)+A \tilde{\gamma}(z(t))+B \tilde{\gamma}(z(t-\tau))+C \int_{t-h}^{t} \tilde{G}(z(s)) d s .
$$

Theorem 4.1. Based on the hypotheses in Theorem 3.1, the equilibrium point of neural network (2) is globally exponentially stable.

Proof. Based on the hypotheses in Theorem 3.1, we choose a sufficiently small constant $\delta>0$ such that

$$
\begin{aligned}
\Gamma= & \left(P A^{*}+A^{* T} P+\left\|P A_{*}+A_{*}^{T} P\right\|_{2} I\right)+\left(1+e^{\delta \tau}\right)\|P\|_{2} \mathrm{~b} \\
& +\|P C\|_{2}^{2} I+e^{\delta \tau} H^{2}<0,
\end{aligned}
$$

and $\delta<\min \left\{\frac{1}{2} d_{i}: 1 \leq i \leq n\right\}$. Consider the following Lyapunov functional

$$
V(t, z(t))=V_{1}(t, z(t))+V_{2}(t, z(t))+V_{3}(t, z(t))+V_{4}(t, z(t)),
$$


where

$$
\begin{aligned}
& V_{1}(t, z(t))=e^{\delta t} z^{T}(t) D^{-1} z(t) \\
& V_{2}(t, z(t))=\alpha e^{\delta t}\left[2 \sum_{i=1}^{n} p_{i} \int_{0}^{z_{i}(t)} \tilde{G}_{i}(\theta) d \theta\right] \\
& \left.V_{3}(t, z(t))=(\alpha \gamma+\beta) \sum_{i=1}^{n} p_{i} \int_{t-\tau_{i}}^{t} \tilde{G}_{i}^{2}(z(s)) e^{\delta\left(s+\tau_{i}\right)} d s\right] \\
& \left.V_{4}(t, z(t))=\sigma \sum_{i=1}^{n} p_{i} \int_{-h_{i}}^{0} \int_{t+\theta}^{t} \tilde{G}_{i}^{2}\left(z_{i}(s)\right) e^{\delta\left(s+\tau_{i}\right)} d s d \theta\right] .
\end{aligned}
$$

Next, we will compute the derivation of $V_{i}(i=1,2,3,4)$ separately. We first compute the derivation of $V_{1}(t, z(t))$ with respect to time along the solution of neural network (18) as follows,

$$
\begin{aligned}
\dot{V}_{1}(t, z(t))= & \delta e^{\delta t} z^{T}(t) D^{-1} z(t)+2 e^{\delta t} z^{T}(t) D^{-1} \dot{z}(t) \\
= & \delta e^{\delta t} z^{T}(t) D^{-1} z(t)-2 e^{\delta t} z^{T}(t) D^{-1} D z(t)+2 e^{\delta t} z^{T}(t) D^{-1} A(z) \tilde{\gamma}(z(t)) \\
& +2 e^{\delta t} z^{T}(t) D^{-1} B(z) \tilde{\gamma}(z(t-\tau))+2 e^{\delta t} z^{T}(t) D^{-1} C \int_{t-h}^{t} g(z(s)) d s \\
\leq & e^{\delta t} z^{T}(t)\left(\delta D^{-1}-\frac{I}{2}\right) z(t)+2 e^{\delta t} \tilde{\gamma}^{T}(z(t))\left\|D^{-1} A\right\|_{2}^{2} \tilde{\gamma}(z(t)) \\
& +2 e^{\delta t} \tilde{\gamma}^{T}(z(t-\tau(t)))\left\|D^{-1} B\right\|_{2}^{2} \tilde{\gamma}(z(t-\tau)) \\
& +2 e^{\delta t} \int_{t-h}^{t} g(z(s)) d s\left\|D^{-1} C\right\|_{2}^{2} \int_{t-h}^{t} g(z(s)) d s \\
\leq & 2 e^{\delta t} \tilde{\gamma}^{T}(z(t))\left\|D^{-1} A\right\|_{2}^{2} \tilde{\gamma}(z(t)) \\
& +2 e^{\delta t} \tilde{\gamma}^{T}(z(t-\tau(t)))\left\|D^{-1} B\right\|_{2}^{2} \tilde{\gamma}(z(t-\tau)) \\
& +2 e^{\delta t} \int_{t-h}^{t} g(z(s)) d s\left\|D^{-1} C\right\|_{2}^{2} \int_{t-h}^{t} g(z(s)) d s .
\end{aligned}
$$


Next, calculate the derivation of $V_{2}(t, z(t))$ along the neural network (18),

$$
\begin{aligned}
\dot{V}_{2}(t, z(t))= & \delta \alpha e^{\delta t}\left[2 \sum_{i=1}^{n} p_{i} \int_{0}^{z_{i}(t)} \tilde{\gamma}_{i}(\theta) d \theta\right]+2 e^{\alpha \delta t} \tilde{\gamma}^{T}(z(t)) P \dot{z}(t) \\
= & 2 \delta \alpha e^{\delta t} \tilde{\gamma}^{T}(z(t)) P z(t)-2 \alpha e^{\delta t} \tilde{\gamma}^{T}(z(t)) P D z(t) \\
& +2 \alpha e^{\delta t} \tilde{\gamma}^{T}(z(t)) P A \tilde{\gamma}(z(t))+2 \alpha e^{\delta t} \tilde{\gamma}^{T}(z(t)) P B \tilde{\gamma}(z(t-\tau)) \\
& +2 \alpha e^{\delta t} \tilde{\gamma}^{T}(z(t)) P C \int_{t-h}^{t} g(z(s)) d s \\
\leq & 2 \alpha e^{\delta t} \tilde{\gamma}^{T}(z(t)) P(\delta I-D) z(t) \\
& +2 \alpha e^{\delta t} \tilde{\gamma}^{T}(z(t)) P A \tilde{\gamma}(z(t))+2 \alpha e^{\delta t} \tilde{\gamma}^{T}(z(t)) P B \tilde{\gamma}(z(t-\tau)) \\
& +\alpha e^{\delta t} \tilde{\gamma}^{T}(z(t))\|P C\|_{2}^{2} \tilde{\gamma}(z(t))+\alpha e^{\delta t} \int_{t-h}^{t} g(z(s)) d s \int_{t-h}^{t} g(z(s)) d s \\
\leq & \alpha e^{\delta t} \tilde{\gamma}^{T}(z(t))\left(P A+A^{T} P\right) \tilde{\gamma}(z(t))+2 \alpha e^{\delta t} \tilde{\gamma}^{T}(z(t)) P B \tilde{\gamma}(z(t-\tau)) \\
& +\alpha e^{\delta t} \tilde{\gamma}^{T}(z(t))\|P C\|_{2}^{2} \tilde{\gamma}(z(t))+\alpha e^{\delta t} \int_{t-h}^{t} g(z(s)) d s \int_{t-h}^{t} g(z(s)) d s .
\end{aligned}
$$

By Lemma 2.1 and Lemma 2.4, we have

(1) $\alpha e^{\delta t} \tilde{\gamma}^{T}(z(t))\left(P A+A^{T} P\right) \tilde{\gamma}(z(t))$

$$
\leq \alpha e^{\delta t} \tilde{\gamma}^{T}(z(t))\left(P A^{*}+A^{* T} P+\left\|P A_{*}+A_{*}^{T} P\right\|_{2} I\right) \tilde{\gamma}(z(t)),
$$

(2) $2 \alpha e^{\delta t} \tilde{\gamma}^{T}(z(t)) P B \tilde{\gamma}(z(t-\tau))$

$$
\begin{aligned}
& \leq 2 \alpha e^{\delta t} \tilde{\gamma}^{T}(z(t))\|P\|_{2} \mathrm{~b} \tilde{\gamma}(z(t-\tau)) \\
& \leq \alpha e^{\delta t} \tilde{\gamma}^{T}(z(t))\|P\|_{2} \mathrm{~b} \tilde{\gamma}(z(t))+\alpha e^{\delta t} \tilde{\gamma}(z(t-\tau))^{T}\|P\|_{2} \mathrm{~b} \tilde{\gamma}(z(t-\tau)) .
\end{aligned}
$$

Substituting (24) into (23), we get

$$
\begin{aligned}
\dot{V}_{2}(t, z(t)) \leq & \alpha e^{\delta t} \tilde{\gamma}^{T}(z(t))\left(P A^{*}+P A^{* T}+\left\|P A_{*}+A_{*}^{T} P\right\|_{2} I\right) \tilde{\gamma}(z(t)) \\
& +\alpha e^{\delta t} \tilde{\gamma}^{T}(z(t))\|P\|_{2} \mathrm{~b} \tilde{\gamma}(z(t)) \\
& +\alpha e^{\delta t} \tilde{\gamma}(z(t-\tau))^{T}\|P\|_{2} \mathrm{~b} \tilde{\gamma}(z(t-\tau)) \\
& +\alpha e^{\delta t} \tilde{\gamma}^{T}(z(t)) P C \tilde{\gamma}(z(t))+\alpha e^{\delta t} \int_{t-h}^{t} g(z(s)) d s P C \int_{t-h}^{t} g(z(s)) d s .
\end{aligned}
$$

The time derivative of $V_{3}(t, z(t))$ along the solution of neural network (18) is obtained as follows

$$
\begin{aligned}
\dot{V}_{3}(t, z(t)) & =(\alpha \gamma+\beta) \sum_{i=1}^{n} p_{i} \int_{t-\tau_{i}}^{t} \tilde{\gamma}_{i}(z(s)) e^{\delta\left(s+\tau_{i}\right)} d s \\
& =(\alpha \gamma+\beta) e^{t+\tau}\|\tilde{\gamma}(z(t))\|_{2}^{2}-(\alpha \gamma+\beta) e^{t}\|\tilde{\gamma}(z(t-\tau))\|_{2}^{2} .
\end{aligned}
$$


The derivation of $V_{4}(t, z(t))$ is shown as folows,

$$
\begin{aligned}
\dot{V}_{4}(t, z(t)) & =\sigma \sum_{i=1}^{n} e^{\delta\left(t+\tau_{i}\right)} p_{i} h_{i}^{2} \tilde{\gamma}_{i}^{2}\left(z_{i}(t)\right)-\sigma \sum_{i=1}^{n} e^{\delta t} p_{i} h_{i}^{2} \int_{t-h_{i}}^{t} \tilde{\gamma}_{i}^{2}\left(z_{i}(s)\right) d s \\
& \leq \sigma e^{\delta(t+\tau)} \tilde{\gamma}^{T}(z(t)) P H^{2} \tilde{\gamma}(z(t))-\sigma e^{\delta t} \int_{t-h}^{t} \tilde{\gamma}(z(s)) d s P \int_{t-h}^{t} \tilde{\gamma}(z(s)) d s .
\end{aligned}
$$

where $H^{2}=\operatorname{diag}\left(h_{1}^{2}, h_{2}^{2}, \ldots, h_{n}^{2}\right)$. Hence, according to the derivation of $\dot{V}_{i}(i=$ $1,2,3,4)$ above, we have

$$
\begin{aligned}
\dot{V}(t, z(t))= & \dot{V}_{1}(t, z(t))+\dot{V}_{2}(t, z(t))+\dot{V}_{3}(t, z(t))+\dot{V}_{4}(t, z(t)) \\
\leq & 2 e^{\delta t} \tilde{\gamma}^{T}(z(t))\left\|D^{-1} A\right\|_{2}^{2} \tilde{\gamma}(z(t))+2 e^{\delta t} \tilde{\gamma}^{T}(z(t-\tau))\left\|D^{-1} B\right\|_{2}^{2} \tilde{\gamma}(z(t-\tau)) \\
& +2 e^{\delta t} \int_{t-h}^{t} g(z(s)) d s\left\|D^{-1} C\right\|_{2}^{2} \int_{t-h}^{t} g(z(s)) d s \\
& +\alpha e^{\delta t} \tilde{\gamma}^{T}(z(t))\left(P A^{*}+A^{* T} P+\left\|P A_{*}+A_{*}^{T} P\right\|_{2} I\right) \tilde{\gamma}(z(t)) \\
& +\alpha e^{\delta t} \tilde{\gamma}^{T}(z(t))\|P\|_{2} \mathrm{~b} \tilde{\gamma}(z(t))+\alpha e^{\delta t} \tilde{\gamma}(z(t-\tau))^{T}\|P\|_{2} \mathrm{~b} \tilde{\gamma}(z(t-\tau)) \\
& +\alpha e^{\delta t} \tilde{\gamma}^{T}(z(t))\|P C\|_{2}^{2} \tilde{\gamma}(z(t))+\alpha e^{\delta t} \int_{t-h}^{t} g(z(s)) d s \int_{t-h}^{t} g(z(s)) d s \\
& +(\alpha \gamma+\beta) e^{\delta(t+\tau)}\|\tilde{\gamma}(z(t))\|_{2}^{2}-(\alpha \gamma+\beta) e^{t}\|\tilde{\gamma}(z(t-\tau))\|_{2}^{2} \\
& +\sigma e^{\delta(t+\tau)} \tilde{\gamma}^{T}(z(t)) P H^{2} \tilde{\gamma}(z(t))-\sigma e^{\delta t} \int_{t-h}^{t} \tilde{\gamma}(z(s)) d s P \int_{t-h}^{t} \tilde{\gamma}(z(s)) d s \\
= & e^{\delta t} \tilde{\gamma}^{T}(z(t))\left[2\left\|D^{-1} A\right\|_{2}^{2} I+\alpha\left(P A^{*}+A^{* T} P+\left\|P A_{*}+A_{*}^{T} P\right\|_{2} I\right)\right. \\
& \left.+\alpha\|P\|_{2} \mathrm{~b} I+(\alpha \gamma+\beta) e^{\tau}+\sigma e^{\delta \tau} P H^{2}+\|P C\|_{2}^{2} I\right] \tilde{\gamma}(z(t)) \\
& +\tilde{\gamma}(z(t-\tau))^{T}\left[2\left\|D^{-1} B\right\|_{2}^{2} I+\alpha\|P\|_{2} \mathrm{~b} I-(\alpha \gamma+\beta) I\right] \tilde{\gamma}(z(t-\tau)) \\
& +\int_{t-h}^{t} \tilde{\gamma}(z(s)) d s\left[2\left\|D^{-1} C\right\|_{2}^{2} I-\sigma P+\alpha I\right] \int_{t-h}^{t} \tilde{\gamma}(z(s)) d s .
\end{aligned}
$$

If we choose

$$
\begin{aligned}
& \beta=2\left\|D^{-1} B\right\|_{2}^{2}, \\
& \gamma=\alpha\|P\|_{2} \mathrm{~b}, \\
& \sigma=\alpha p_{m}^{-1}+2 p_{m}^{-1}\left\|D^{-1} C\right\|_{2}^{2},
\end{aligned}
$$


where $p_{m}=\min _{i=1, \ldots, n} p_{i}$. Then, $\dot{V}(t, z(t))$ can be written as

$$
\begin{aligned}
& \dot{V}(t, z(t)) \leq \alpha e^{\delta t} \tilde{\gamma}^{T}(z(t))\left[\left(P A^{*}+A^{* T} P+\left\|P A_{*}+A_{*}^{T} P\right\|_{2} I\right)+\left(1+e^{\delta \tau}\right)\|P\|_{2} \mathrm{~b} I\right. \\
&\left.+\|P C\|_{2}^{2} I+e^{\delta \tau} H^{2}\right] \tilde{\gamma}(z(t)) \\
&+e^{\delta t} \tilde{\gamma}^{T}(z(t))\left[2\left\|D^{-1} A\right\|_{2}^{2} I+2 e^{\delta \tau}\left\|D^{-1} B\right\|_{2}^{2} I\right. \\
&\left.+2 e^{\delta \tau}\left\|D^{-1} C\right\|_{2}^{2} H^{2}\right] \tilde{\gamma}(z(t)) \\
&=e^{\delta t} \tilde{\gamma}^{T}(z(t))\left[\alpha \Gamma+2\left\|D^{-1} A\right\|_{2}^{2} I+2 e^{\delta \tau}\left\|D^{-1} B\right\|_{2}^{2} I\right. \\
&\left.+2 e^{\delta \tau}\left\|D^{-1} C\right\|_{2}^{2} H^{2}\right] \tilde{\gamma}(z(t))
\end{aligned}
$$

Since $\Gamma$ from (20) is a negative define matrix, we can choose a sufficient large $\alpha$ such that

$$
\alpha \Gamma+2\left\|D^{-1} A\right\|_{2}^{2} I+2 e^{\delta \tau}\left\|D^{-1} B\right\|_{2}^{2} I+2 e^{\delta \tau}\left\|D^{-1} C\right\|_{2}^{2} H^{2}<0,
$$

which implies,

$$
\dot{V}(t, z(t)) \leq 0 .
$$

Hence, $V(t, z(t)) \leq V(0, z(0))$, i.e.,

$$
V_{1}(t, z(t)) \leq V(t, z(t)) \leq V(0, z(0)),
$$

which means

$$
e^{\delta t} z^{T}(t) D^{-1} z(t) \leq V(0, z(0))
$$

By (32), we obtain

$$
z^{T}(t) z(t) \leq V(0, z(0)) e^{-\delta t} D_{M},
$$

where $D_{M}=\max \left\{d_{i}\right\}$.

$$
\left|x(t)-x^{*}\right| \leq D_{M}[V(0, z(0))]^{\frac{1}{2}} e^{-\frac{\delta t}{2}} .
$$

This means that neural network (2) is globally robustly exponentially stable. Moreover, it also means that the equilibrium point of neural network (2) is unique.

\section{Comparisons and examples}

In this section, by making further analysis and giving some numerical examples, we compare our results with the previous ones derived in the previous literatures. We first compare our results with the results in [32]. The neural network considered in [32] is

$$
\dot{x}(t)=-D x(t)+A g(x(t))+B g(x(t-\tau))+U .
$$

Obviously, the neural network (33) considered in [32] is a special case of the neural network (2) in this paper. Moreover, to some extent, the results in this paper improve the results in [32]. The following example explains it. 
Example 1. we consider a delayed neural network model (2) with the following network parameters:

$$
\begin{aligned}
& \bar{A}=\left(\begin{array}{cc}
-107 & 0 \\
0 & -103
\end{array}\right), \underline{A}=\left(\begin{array}{cc}
-111 & 0 \\
0 & -107
\end{array}\right), \bar{B}=\left(\begin{array}{cc}
100 & 0 \\
0 & 100
\end{array}\right) \\
& \underline{B}=\left(\begin{array}{cc}
85 & 0 \\
0 & 70
\end{array}\right), \bar{C}=\left(\begin{array}{cc}
1 & 0 \\
0 & 0.9
\end{array}\right), \underline{C}=\left(\begin{array}{cc}
0.5 & 0 \\
0 & 0.4
\end{array}\right), \bar{D}=\left(\begin{array}{cc}
10 & 0 \\
0 & 12
\end{array}\right) \\
& \underline{D}=\left(\begin{array}{cc}
9 & 0 \\
0 & 11
\end{array}\right), H=\left(\begin{array}{cc}
1 & 0 \\
0 & 1
\end{array}\right), K=\left(\begin{array}{cc}
2 & 0 \\
0 & 3
\end{array}\right), g(x)=\left(\begin{array}{c}
\frac{1}{3} x \\
\frac{1}{4} x
\end{array}\right), U=(1,1) \\
& \tau=1
\end{aligned}
$$

Obviously, for any positive diagonal matrix $P$,

$$
\left(\begin{array}{cc}
-P \tilde{A}-\tilde{A}^{T} P-\left(\left\|B^{*}\right\|+\left\|B_{*}\right\|\right)^{2} I_{n} & -P \\
-P & I_{n}
\end{array}\right)
$$

is not a positive definite matrix. Hence, The results in ([32]) cannot be used to verify the robust stability of the defined neural network. Here we use Theorem 4.1 to verify the global robust exponential stability of the defined neural network in the example. Firstly, it is obvious that $g$ satisfy hypotheses $\left(\mathbf{A}_{\mathbf{1}}, \mathbf{A}_{\mathbf{2}}\right)$. After simple calculation, $\mathrm{b}=4$. Then, if we choose $P=\left(\begin{array}{ll}1 & 0 \\ 0 & 1\end{array}\right)$, we have

$$
\Phi_{1}=\left(\begin{array}{cc}
-12.95 & 0 \\
0 & -4.95
\end{array}\right)<0
$$

Hence, by Theorem 3.1 and Theorem 4.1, the defined neural network in the example has an equilibrium point, which is globally robustly exponentially stable. To test and verify the theoretical result, we give some simulations by the following cases:

Case 1. $A=\bar{A}, B=\bar{B}, C=\bar{C}, D=\bar{D}$, see Figure 1 .

Case 2. $A=\left(\begin{array}{cc}109 & 0.1 \\ 0.1 & 105\end{array}\right) \in A_{I}, B=\left(\begin{array}{cc}90 & 0 \\ 0 & 85\end{array}\right) \in B_{I}, C=\left(\begin{array}{cc}0.7 & 0 \\ 0 & 0.6\end{array}\right) \in$ $C_{I}, D=\left(\begin{array}{cc}9.5 & 0 \\ 0 & 11.5\end{array}\right) \in D_{I}$, see Figure 1 .

The following example is used to show the advantage of this paper compared with Theorem 3 in [40]. 

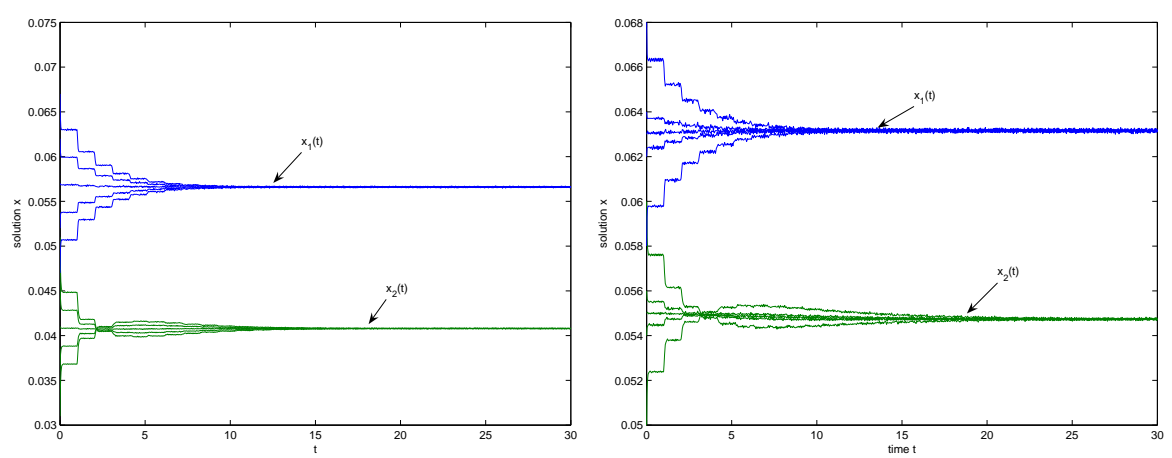

Figure 1: Trajectories of neural network in case 1 and 2 of Example 1 with five different initial points.

Example 2. We consider a delayed neural network model (2) with the following network parameters:

$$
\begin{aligned}
& \bar{A}=\left(\begin{array}{cc}
-6 & 0 \\
0 & -5
\end{array}\right), \underline{A}=\left(\begin{array}{cc}
-8 & 0 \\
0 & -7
\end{array}\right), \bar{B}=\left(\begin{array}{cc}
5 & 0 \\
0 & 3
\end{array}\right), \underline{B}=\left(\begin{array}{ll}
4 & 0 \\
0 & 2
\end{array}\right) \\
& \bar{C}=\left(\begin{array}{cc}
-1 & 0 \\
0 & -0.5
\end{array}\right), \underline{C}=\left(\begin{array}{cc}
-2 & 0 \\
0 & -1
\end{array}\right), \bar{D}=\left(\begin{array}{cc}
10 & 0 \\
0 & 12
\end{array}\right), \underline{D}=\left(\begin{array}{cc}
9 & 0 \\
0 & 11
\end{array}\right) \\
& H=\left(\begin{array}{cc}
1 & 0 \\
0 & 1
\end{array}\right), K=\left(\begin{array}{cc}
\frac{1}{2} & 0 \\
0 & 1
\end{array}\right), g(x)=\left(\begin{array}{c}
\frac{1}{3} x \\
\frac{1}{4} x
\end{array}\right), U=(1,1), \tau=1
\end{aligned}
$$

Obviously, for any positive diagonal matrix $P$,

$$
\begin{aligned}
\Phi_{4} & =2 \underline{C} P K^{-1}-\left(P A^{*}+A^{* T} P+\left\|P A^{*}+A_{*}^{T} P\right\| I\right)-2\|P\|_{2}\|Q\|_{2} I \\
& =\left(\begin{array}{cc}
6 p_{1}-10.809 p-2 & 0 \\
0 & 10 p_{2}-10,809 p-2
\end{array}\right)
\end{aligned}
$$

is not a positive definite matrix, where $p=\max \left\{p_{1}, p_{2}\right\}$. Hence, Theorem 3 in [40] cannot be used to verify the robust stability of the defined neural network. Here we use Theorem 4.1 to verify the global robust exponential stability of the defined neural network in the example. Firstly, it is obvious that g satisfy hypotheses $\left(\mathbf{A}_{\mathbf{1}}, \mathbf{A}_{\mathbf{2}}\right)$. After simple calculation, $\mathrm{b}=4$. Then, if we choose $P=\left(\begin{array}{cc}1 & 0 \\ 0 & 1\end{array}\right)$, we have $\Phi_{1}=\left(\begin{array}{cc}-2.188 & 0 \\ 0 & -0.188\end{array}\right)<0$.

Hence, by Theorem 3.1 and Theorem 4.1, the defined neural network in the example has an equilibrium point, which is globally robustly exponentially stable. To test and verify the theoretical result, we give some simulations by the following cases:

Case 3. $A=\bar{A}, B=\bar{B}, C=\bar{C}, D=\bar{D}$, see Figure 2. 
Case 4. $A=\left(\begin{array}{cc}-7 & 0 \\ 0 & -6\end{array}\right) \in A_{I}, B=\left(\begin{array}{cc}4.5 & 0 \\ 0 & 2.5\end{array}\right) \in B_{I}, C=\left(\begin{array}{cc}-1.5 & 0 \\ 0 & -0.7\end{array}\right) \in$ $C_{I}, D=\left(\begin{array}{cc}9.5 & 0 \\ 0 & 11.5\end{array}\right) \in D_{I}$, see Figure 2.
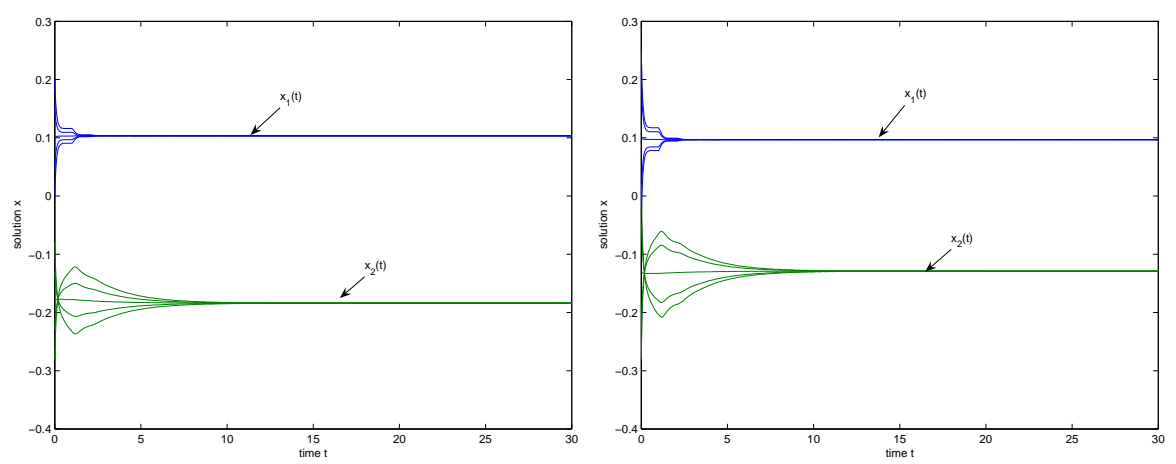

Figure 2: Trajectories of neural network in case 3 and 4 of Example 2 with five different initial points.

Example 3. We consider a delayed neural network model (2) with the following network parameters:

$$
\begin{aligned}
& \bar{A}=\left(\begin{array}{cc}
-26 & 0 \\
0 & -28
\end{array}\right), \underline{A}=\left(\begin{array}{cc}
-30 & 0 \\
0 & -32
\end{array}\right), \bar{B}=\left(\begin{array}{cc}
20 & 0 \\
0 & 19
\end{array}\right), \underline{B}=\left(\begin{array}{cc}
16 & 0 \\
0 & 11
\end{array}\right) \\
& \bar{C}=\left(\begin{array}{cc}
3 & 0.01 \\
0.01 & 2
\end{array}\right), \underline{C}=\left(\begin{array}{cc}
2 & 0.01 \\
0.01 & 1
\end{array}\right), \bar{D}=\left(\begin{array}{cc}
10 & 0 \\
0 & 12
\end{array}\right), \underline{D}=\left(\begin{array}{cc}
9 & 0 \\
0 & 11
\end{array}\right) \\
& H=\left(\begin{array}{ll}
1 & 0 \\
0 & 1
\end{array}\right), K=\left(\begin{array}{cc}
\frac{1}{2} & 0 \\
0 & 1
\end{array}\right), g(x)=\left(\begin{array}{c}
\frac{1}{3} x \\
\frac{1}{4} x
\end{array}\right), U=(1,1), \tau=1
\end{aligned}
$$

After simple calculation, for any positive diagonal matrix $P$,

$$
2 P C K^{-1}-\left(P A^{*}+A^{* T} P+\left\|P A^{*}+A_{*}^{T} P\right\| I\right)-L-\frac{1}{1-h} b_{1}\|P\|_{2}\|L\|_{2} I
$$

is not a positive definite matrix. Hence, Theorem 3 in [42] cannot be used to verify the robust stability of the defined neural network. On the other hand, if we choose $P=\left(\begin{array}{ll}1 & 0 \\ 0 & 1\end{array}\right)$, we have $\Phi_{1}=\left(\begin{array}{cc}-10.9973 & 0 \\ 0 & -14.9973\end{array}\right)<0$.

Hence, by Theorem 3.1 and Theorem 4.1, the defined neural network in the example has an equilibrium point, which is globally robustly exponentially stable. For details, see case 4 and Figure 3 below. To test and verify the theoretical result, we give some simulations by the following cases: 
Case 5. $A=\bar{A}, B=\bar{B}, C=\bar{C}, D=\bar{D}$, see Figure 3 .

Case 6. $A=\left(\begin{array}{cc}-28 & 0 \\ 0 & -30\end{array}\right) \in A_{I}, B=\left(\begin{array}{cc}18 & 0 \\ 0 & 15\end{array}\right) \in B_{I}, C=\left(\begin{array}{cc}2.5 & 0.01 \\ 0.01 & 1.5\end{array}\right) \in$ $C_{I}, D=\left(\begin{array}{cc}9.5 & 0 \\ 0 & 11.5\end{array}\right) \in D_{I}$, see Figure 3.
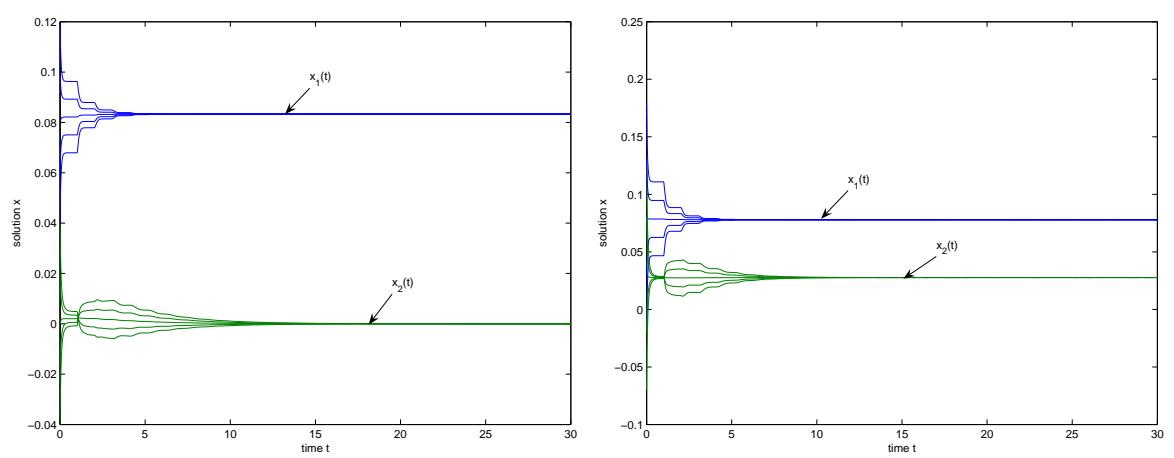

Figure 3: Trajectories of neural network in case 5 and 6 of Example 3 with five different initial points.

Example 4. We consider the delayed neural network (2) with the following network parameters:

$$
\begin{aligned}
& \bar{A}=\left(\begin{array}{cc}
-26 & 0 \\
0 & -28
\end{array}\right), \underline{A}=\left(\begin{array}{cc}
-30 & 0 \\
0 & -32
\end{array}\right), \bar{B}=\left(\begin{array}{cc}
20 & 0 \\
0 & 19
\end{array}\right), \underline{B}=\left(\begin{array}{cc}
16 & 0 \\
0 & 11
\end{array}\right) \\
& \bar{C}=\left(\begin{array}{cc}
3 & 0.01 \\
0.01 & 2
\end{array}\right), \underline{C}=\left(\begin{array}{cc}
2 & 0.01 \\
0.01 & 1
\end{array}\right), \bar{D}=\left(\begin{array}{cc}
10 & 0 \\
0 & 12
\end{array}\right), \underline{D}=\left(\begin{array}{cc}
9 & 0 \\
0 & 11
\end{array}\right) \\
& H=\left(\begin{array}{ll}
1 & 0 \\
0 & 1
\end{array}\right), K=\left(\begin{array}{cc}
2 & 0 \\
0 & 3
\end{array}\right), g(x)=\left(\begin{array}{l}
g_{1}(x) \\
g_{2}(x)
\end{array}\right), U=(1,1), \tau=1
\end{aligned}
$$

where

$$
g_{1}(x)=g_{2}(x)= \begin{cases}x+1 & x>1 \\ x & 1 \geq x\end{cases}
$$

Obviously, for any positive diagonal matrix $P$,

$$
\begin{aligned}
\Phi_{5} & =2 P C K^{-1}-\left(P A^{*}+A^{* T} P+\left\|P A^{*}+A_{*}^{T} P\right\| I\right)-L-\frac{1}{1-h} b_{1}\|P\|_{2}\|L\|_{2} I \\
& =\left(\begin{array}{cc}
\Delta_{1} & 0 \\
0 & \Delta_{2}
\end{array}\right)
\end{aligned}
$$


where $\Delta_{1}=68 p_{1}-\left\|\begin{array}{cc}4 p_{1} & 0 \\ 0 & 4 p_{2}\end{array}\right\|_{2}-l_{1}-400.004 * \frac{1}{1-h}\|P\|_{2}^{2}\left\|L^{-1}\right\|_{2}$, $\Delta_{2}=66 p_{2}-\left\|\begin{array}{cc}4 p_{1} & 0 \\ 0 & 4 p_{2}\end{array}\right\|_{2}-l_{2}-400.004 * \frac{1}{1-h}\|P\|_{2}^{2}\left\|L^{-1}\right\|_{2}$.

Since $0 \leq l_{i} \leq 1,0 \leq h \leq 1$,

$\Phi_{5} \leq\left(\begin{array}{cc}68 p_{1}-4\|P\|_{2}-400.004\|P\|_{2}^{2} & 0 \\ 0 & 66 p_{2}-4\|P\|_{2}-400.004\|P\|_{2}^{2}\end{array}\right)$

is not a positive definite matrix, where $p=\max \left\{p_{1}, p_{2}\right\}$. Hence, Theorem 3 in [43] cannot be used to verify the robust stability of the defined neural network. Here we use Theorem 4.1 to verify the global robust exponential stability of the defined neural network in the example. Firstly, it is obvious that $g$ satisfy hypotheses $\left(\mathbf{A}_{\mathbf{1}}, \mathbf{A}_{\mathbf{2}}\right)$. After simple calculation, $\mathrm{b}=4$. Then, if we choose $P=\left(\begin{array}{ll}1 & 0 \\ 0 & 1\end{array}\right)$, we have

$$
\Phi_{1}=\left(\begin{array}{cc}
-10.9973 & 0 \\
0 & -14.9973
\end{array}\right)<0
$$

Hence, by Theorem 3.1 and Theorem 4.1, the defined neural network in the example has an equilibrium point, which is globally robustly exponentially stable. To test and verify the theoretical result, we give some simulations by the following cases:

Case 7. $A=\bar{A}, B=\bar{B}, C=\bar{C}, D=\bar{D}$, see Figure 4.

Case 8. $A=\left(\begin{array}{cc}-28 & 0 \\ 0 & -30\end{array}\right) \in A_{I}, B=\left(\begin{array}{cc}18 & 0 \\ 0 & 15\end{array}\right) \in B_{I}, C=\left(\begin{array}{cc}2.5 & 0.01 \\ 0.01 & 1.5\end{array}\right) \in$ $C_{I}, D=\left(\begin{array}{cc}9.5 & 0 \\ 0 & 11.5\end{array}\right) \in D_{I}$, see Figure 4.

\section{Conclusions}

In this paper, we study the global exponential stability of a class of neural networks with discontinuous Lurie-type activation and mixed delays. We propose a new sufficient condition for the existence and global exponential stability of the equilibrium point under parameter uncertainties of the neural network. We make a detailed comparison between our result and the previous corresponding results derived in the previous literature. These comparisons show that our result is new and improves some of the previously reported stability results. 

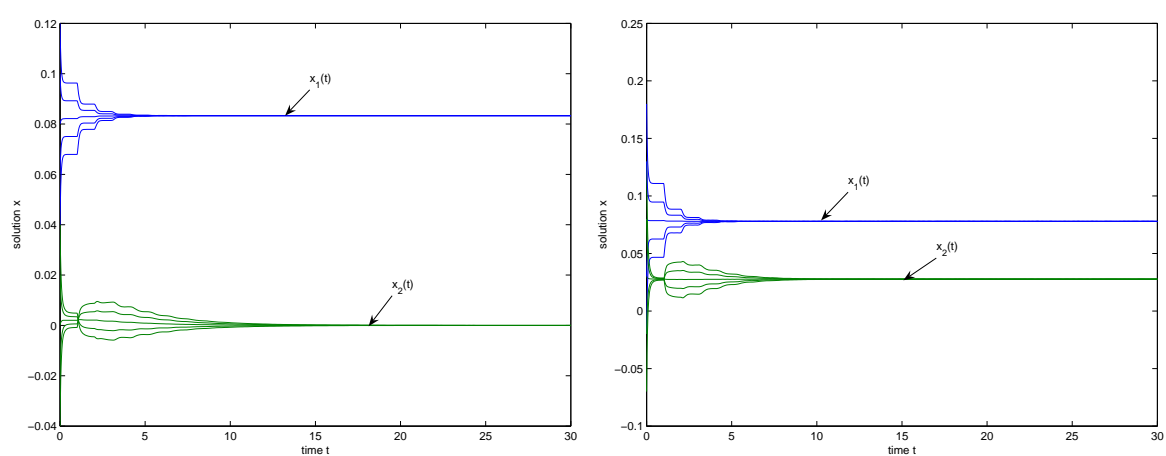

Figure 4: Trajectories of neural network in case 7 and 8 of Example 4 with five different initial points.

\section{References}

[1] X. B. Gao, A novel neural network for nonlinear convex programming, IEEE Trans. Neural Networks 15 (3) (2004) 613-621.

[2] Y. Zhang, J. Wang, Y. Xia, A dual neural network for redundancy resolution of kinematically redundant manipulators subject to joint limits and joint velocity limits, IEEE Trans. Neural Networks 14 (3) (May 2003) 658667.

[3] Q. Liu, J. Cao, G. Chen, A novel recurrent neural network with finite-time convergence for linear programming, Neural Computation 22 (11) (2010) 2962-2978.

[4] Q. Liu, J. Wang, Finite-time convergent recurrent neural network with a hard-limiting activation function for constrained optimization with piecewise-linear objective functions, IEEE Trans. Neural Networks 22 (4) (Apr. 2011) 601-613.

[5] S. Qin, W. Bian, X. Xue, A new one-layer recurrent neural network for nonsmooth pseudoconvex optimization, Neurocomputing 120 (2013) 655662 .

[6] S. Qin, X. Xue, Dynamical behavior of a class of nonsmooth gradient-like systems, Neurocomputing 73 (13-15) (2010) 2632-2641.

[7] Y. Liao, S. C. Fang, H. L. W. Nuttle, A neural network model with bounded-weights for pattern classification, Computers and Operations Research 31 (9) (2004) 1411-1426.

[8] A. Wu, S. Wen, Z. Zeng, Synchronization control of a class of memristorbased recurrent neural networks, Information Sciences 183 (1) (2012) 106116. 
[9] O. Faydasicok, S. Arik, Equilibrium and stability analysis of delayed neural networks under parameter uncertainties, Applied Mathematics and Computation 218 (12) (2012) 6716-6726.

[10] O. Faydasicok, S. Arik, A new robust stability criterion for dynamical neural networks with multiple time delays, Neurocomputing 99 (2013) 290-297.

[11] A. Wu, Z. Zeng, Dynamic behaviors of memristor-based recurrent neural networks with time-varying delays, Neural Networks 36 (2012) 1-10.

[12] S. Wen, Z. Zeng, T. Huang, Adaptive synchronization of memristor-based chua s circuits, Physics Letters A 376 (44) (2012) 2775-2780.

[13] S. Arik, A new condition for robust stability of uncertain neural networks with time delays, Neurocomputing 128 (2014) 476-482.

[14] S. Arik, An improved robust stability result for uncertain neural networks with multiple time delays, Neural Networks 54 (2014) 1-10.

[15] S. Arik, New criteria for global robust stability of delayed neural network$\mathrm{s}$ with norm-bounded uncertainties, IEEE Trans. Neural Netw. Learning Syst. 25 (6) (2014) 1045-1052.

[16] S. Qin, J. Xu, X. Shi, Convergence analysis for second-order interval cohengrossberg neural networks, Communications in Nonlinear Science and Numerical Simulation 19 (8) (2014) 2747-2757.

[17] S. Qin, X. Xue, P. Wang, Global exponential stability of almost periodic solution of delayed neural networks with discontinuous activations, Information Sciences 220 (2013) 367-378.

[18] S. Qin, X. Xue, Global exponential stability and global convergence in finite time of neural networks with discontinuous activations, Neural Processing Letters 29 (3) (2009) 189-204.

[19] Z. Cai, L. Huang, Z. Guo, X. Chen, On the periodic dynamics of a class of time-varying delayed neural networks via differential inclusions, Neural Networks 33 (2012) 97-113.

[20] Y. Wang, L. Huang, Dynamical behaviors of cohen-grossberg neural networks with mixed time delays and discontinuous activations, Neurocomputing 121 (2013) 379-386.

[21] X. Chen, L. Huang, Z. Guo, Finite time stability of periodic solution for hopfield neural networks with discontinuous activations, Neurocomputing 103 (2013) 43-49.

[22] L. Duan, L. Huang, Global exponential stability of fuzzy bam neural networks with distributed delays and time-varying delays in the leakage terms, Neural Computing and Applications 23 (Supplement-1) (2013) 171-178. 
[23] L. Duan, L. Huang, Global dynamics of equilibrium point for delayed competitive neural networks with different time scales and discontinuous activations, Neurocomputing 123 (2014) 318-327.

[24] L. Duan, L. Huang, Z. Cai, Existence and stability of periodic solution for mixed time-varying delayed neural networks with discontinuous activations, Neurocomputing 123 (2014) 255-265.

[25] Z. Quan, L. Huang, S. Yu, Z. Zhang, Novel lmi-based condition on global asymptotic stability for bam neural networks with reaction-diffusion terms and distributed delays, Neurocomputing 136 (2014) 213-223.

[26] Z. Guo, J. Wang, Z. Yan, A systematic method for analyzing robust stability of interval neural networks with time-delays based on stability criteria, Neural Networks 54 (2014) 112-122.

[27] E. Y. Mustafa Sayli, Global robust asymptotic stability of variable-time impulsive bam neural networks, Neural Networks 60 (2014) 67-73.

[28] S. Qin, D. Fan, M. Yan, Q. Liu, Global robust exponential stability for interval delayed neural networks with possibly unbounded activation functions, Neural processing letters 40 (1) (2014) 35-50.

[29] S. Wen, G. Bao, Z. Zeng, Y. Chen, T. Huang, Global exponential synchronization of memristor-based recurrent neural networks with time-varying delays, Neural Networks 48 (2013) 195-203.

[30] Z. Zeng, W. X. Zheng, Multistability of neural networks with time-varying delays and concave-convex characteristics, Neural Networks and Learning Systems, IEEE Transactions on 23 (2) (2012) 293-305.

[31] L. Huang, Z. Cai, L. Zhang, L. Duan, Dynamical behaviors for discontinuous and delayed neural networks in the framework of filippov differential inclusions, Neural Networks 48 (2013) 180-194.

[32] Z. Guo, L. Huang, LMI conditions for global robust stability of delayed neural networks with discontinuous neuron activations, Applied Mathematics and Computation 215 (3) (2009) 889-900.

[33] Z. Zeng, Y. Huang, W. X. Zheng, Multistability of recurrent neural networks with time-varying delays and the piecewise linear activation function, Neural Networks, IEEE Transactions on 21 (8) (2010) 1371-1377.

[34] S. Wen, Z. Zeng, T. Huang, Exponential stability analysis of memristorbased recurrent neural networks with time-varying delays, Neurocomputing 97 (2012) 233-240.

[35] M. Forti, P. Nistri, D. Papini, Global exponential stability and global convergence in finite time of delayed neural networks with infinite gain, IEEE Trans. Neural Netw. 16 (6) (2005) 1449-1463. 
[36] M. Forti, P. Nistri, Global convergence of neural networks with discontinuous neuron activations, IEEE Trans. Circuits Syst. I Regul.Pap. 50 (2003) $1421-1435$.

[37] J. Xiao, Z. Zeng, W. Shen, Global asymptotic stability of delayed neural networks with discontinuous neuron activations, Neurocomputing 118 (2013) 322-328.

[38] Y. Li, H. Wu, Global stability analysis in cohen-grossberg neural networks with delays and inverse hölder neuron activation functions, Inf. Sci. 180 (20) (2010) 4022-4030.

[39] A. Granas, J. Dugundji, Fixed point theory, Springer Science \& Business Media, 2003.

[40] O. Faydasicok, S. Arik, Robust stability analysis of a class of neural networks with discrete time delays, Neural Networks 29-30 (2012) 52-59.

[41] G. K, An integral inequality in the stability problem of time-delay systems, Decision and Control, 2000. Proceedings of the 39th IEEE Conference on 3 (2000) 2805-2810.

[42] J.-L. Shao, T.-Z. Huang, S. Zhou, Some improved criteria for global robust exponential stability of neural networks with time-varying delays, Communications in Nonlinear Science and Numerical Simulation 15 (12) (2010) $3782-3794$.

[43] Y. Li, H. Wu, Global stability analysis in cohen-grossberg neural networks with delays and inverse hölder neuron activation functions, Inf. Sci. 180 (20) (2010) 4022-4030. 


\section{Biography of the author(s)}

1. Sitian Qin qinsitian@163.com,

Department of Mathematics, Harbin Institute of Technology at Weihai, Weihai 264209, PR China.

2. Qun Cheng chengqunchn@163.com,

Department of Mathematics, Harbin Institute of Technology at Weihai, Weihai 264209, PR China.

3. Guofang Chen hitbri@126.com,

Department of Civil Engineering, Harbin Institute of Technology at Weihai, Weihai 264209, PR China 


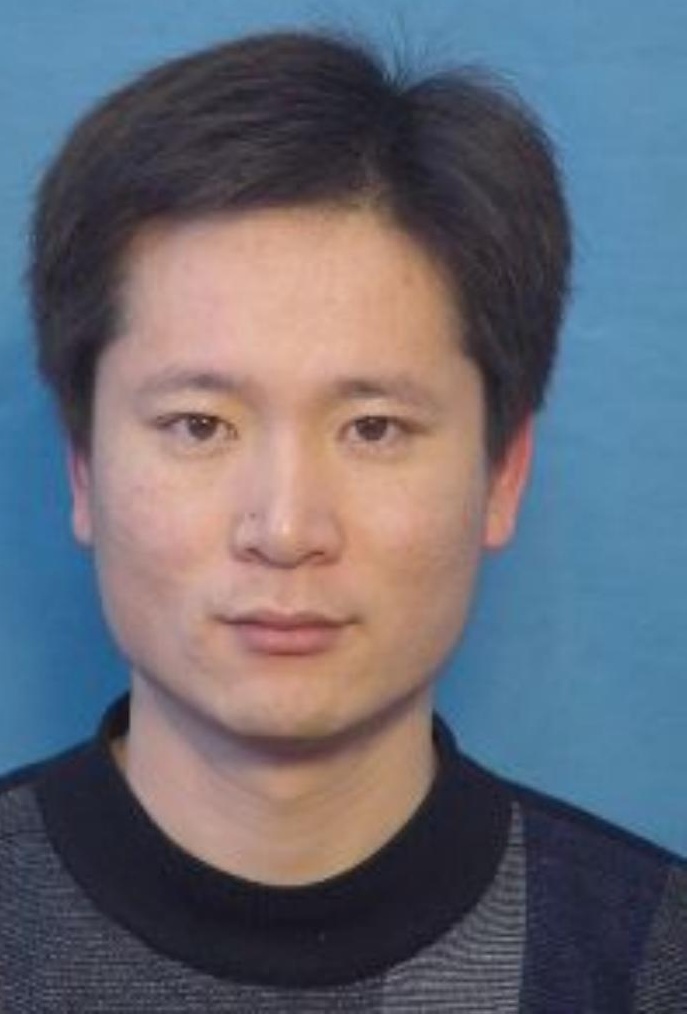

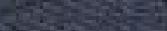

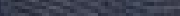

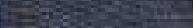

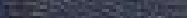

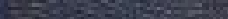

$x=2$

$\cos$

2x:

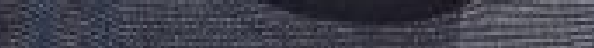




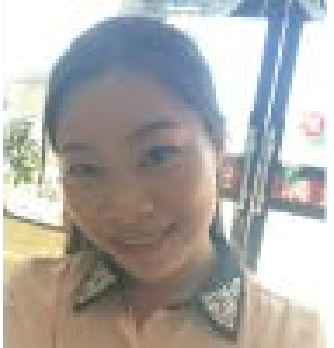




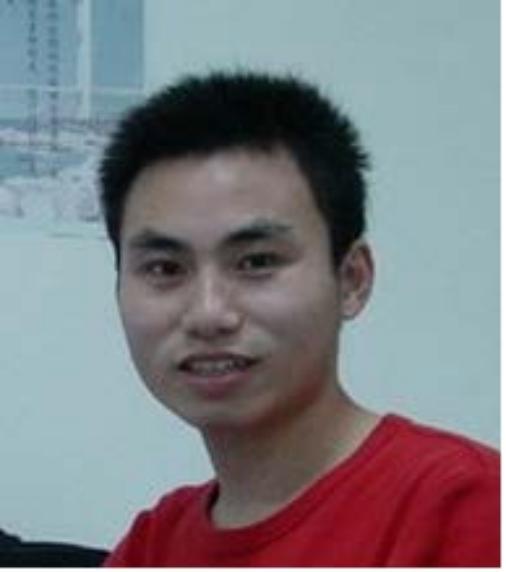

